\title{
Reflexión de Buenas Prácticas Docentes como eje de Calidad en la Educación Universitaria: Caso Escuela de Ciencias Empresariales de la Universidad Católica del Norte
}

\author{
Luz M. Yáñez-Galleguillos ${ }^{(1)}$ y Karla Soria-Barreto ${ }^{(2) *}$ \\ Universidad Católica del Norte, (1) Centro de Innovación Metodológica y Tecnológica, (2) Escuela de \\ Ciencias Empresariales, Larrondo 1281, Coquimbo-Chile (e-mail: Imyanez@ucn.cl; ksoria@ucn.cl) \\ * Autor a quien debe ser dirigida la correspondencia
}

Recibido Dic. 16, 2016; Aceptado Feb. 27, 2017; Versión final Abr. 11, 2017, Publicado Oct. 2017

\begin{abstract}
Resumen
El artículo muestra los resultados del estudio cuyo objetivo fue determinar factores que favorecen el desarrollo de buenas prácticas de docentes de educación superior en dos carreras del área de gestión en una universidad chilena. El tipo de investigación es cualitativo y la técnica utilizada para motivar la reflexión de los dos agentes partícipes (docentes y estudiantes) fue mediante grupos focales. El trabajo de campo consistió en describir, desde la perspectiva de los sujetos, su opinión y reflexión sobre las prácticas docentes en el aula. Se realizaron tres sesiones, dos de estudiantes y una de docentes, con una participación total de 17 estudiantes y 6 docentes. Los resultados detectan ocho factores que potencian las buenas prácticas docentes y cinco de ellos se relacionan con el modo de suministro de la información. Los docentes poseen actitudes positivas hacia la docencia vinculada a su entorno familiar. Para los estudiantes, lo trascendental para lograr sus aprendizajes es la diversidad de metodologías experimentadas en el aula y el perfil del profesor.
\end{abstract}

Palabra clave: buenas prácticas docentes; calidad en la educación; docente; enseñanza-aprendizaje

\section{Reflection of Good Teaching Practices as axis of Quality in University Education: Case of the UCN School of Business}

\begin{abstract}
This article shows the results of the study whose objective was to determine those factors that favor the development of good practices of higher education teachers in two management careers of a Chilean university. The type of research is qualitative and the techniques used to motivate the reflection of the two participating agents (teachers and students) were through focus groups. The field work consisted in describing, from the perspective of the subjects, their opinion and reflection on the teaching practices in the classroom. Three sessions were held, two of students and one of teachers, with a total participation of 17 students and 6 teachers. The results detect eight factors that promote good teaching practices and five of them relate to the way in which information is delivered. Teachers have positive attitudes toward teaching linked to their family environment. For students, a transcendental factor to achieve their learning are the diversity of methodologies experienced in the classroom and the profile of the teacher.
\end{abstract}

Keywords: best teaching practices; quality of education; teacher; teaching learning 


\section{INTRODUCCIÓN}

El desarrollo y progreso de los países va directamente relacionado con la educación de la población. Dentro de los diferentes niveles del sistema educativo, la educación superior es un factor distintivo en relación a los ingresos promedio de las personas, es decir, a mayor nivel educacional mayor ingreso promedio. Un tema que ha cobrado relevancia los últimos años, en todos los niveles del sistema educativo, pero con mayor énfasis en la educación superior, ha sido velar por la calidad de ésta. Para lograr una educación de calidad, se deben tener presente, muchos y variados ámbitos de acción, tales como: docentes, procesos, infraestructura, financiamiento, por nombrar algunos, las cuales intervienen en el análisis profundo de la problemática (González et al. 2012). Quizás aquel que mayor atención concentra es el tema de la docencia y práctica en el aula, lo que se vincula directamente con las buenas prácticas docentes (BPD).

Si hablamos de docencia y su calidad, en el ámbito universitario, es imprescindible analizar la capacidad reflexiva, de quienes ejercen la docencia. Esta herramienta permite establecer mejoras en su actividad docente, como asimismo, el desarrollo y potenciación de habilidades de motivación y facilitación del proceso de enseñanza-aprendizaje. A partir de la reflexión, los docentes pueden ser capaces de seleccionar las estrategias y procedimientos más pertinentes en la práctica de la enseñanza y de instalar mecanismos de evaluación abiertos, profundos y consistentes con las experiencias de aprendizaje. Lo anterior, cobra relevancia si consideramos que el perfil de los estudiantes está cambiando; que surgen nuevos soportes tecnológicos y modelos educativos (digitales), se dan nuevos modos de relación y comunicación entre estudiantes y profesores, por citar algunos (Vásquez, 2015).

En las últimas dos décadas, la educación superior chilena ha experimentado un aumento importante en el número de estudiantes, así como un rápido crecimiento en la variedad de instituciones y programas que se ofrecen. Como señalan Rubio et al. (2016), un ámbito de investigación que se ha explorado poco, tiene que ver con la percepción sobre las actividades concretas de aprendizaje para el desarrollo de competencias. En esa línea de trabajo, el aporte de esta investigación radica en poder ampliar al conocimiento de buenas prácticas docentes en el área de gestión de empresas por medio de la identificación, selección y visualización de los factores que potencian las BPD en una universidad chilena. Estos tres aspectos contribuyen al fortalecimiento de una mejor educación a través de mejores procesos de enseñanzaaprendizaje (Zabalza, 2012). Se sabe que las buenas prácticas docentes se relacionan estrechamente con su contexto, por lo tanto, este estudio se aventura a ver si existe coincidencia con resultados previos en otras áreas del conocimiento y países. En este caso la investigación se realizó tanto en docentes como estudiantes de dos carreras vinculadas a la gestión de empresas en la Universidad Católica del Norte con sede en la ciudad de Coquimbo, ubicada al norte de Chile.

\section{La educación superior en Chile}

En 1981, se estructura un marco regulatorio para la creación de nuevas instituciones de educación superior, dando espacio al aporte privado en su constitución, cuyo ejemplo más palpable, fueron los Institutos Profesionales y Centros de Formación Técnica de carácter privado. Ya en los años '90, la regulación jurídica, incidió en el ingreso al sistema de educación superior de nuevas instituciones, desarrollando un proceso de Licenciamiento en cada una de ellas, para el otorgamiento de su propia autonomía, proceso gestionado, entonces, por el Consejo Superior de Educación, actualmente Consejo Nacional de Educación. (SIES, 2012).

El total de estudiantes matriculados en la educación superior ha tenido un crecimiento sustancial. El año 2002 ascendía a 520.000 jóvenes y diez años después, esta cifra se vio incrementada en más del 100\%, llegando a 1.100 .000 estudiantes aproximadamente. Los alumnos poseen claras diferencias sociales y económicas; destaca el hecho que tienen diversas características en su ingreso y formación previa. Esto, ha generado una variedad de oferta de programas para las distintas necesidades formativas. Como dato adicional, desde el año 2007 al 2016, el 42\% de los estudiantes que ingresan a las universidades tradicionales son la primera generación familiar en ingresar a la educación superior. Esto diversifica el perfil de los estudiantes universitarios, lo que implica nuevos escenarios a tomar en cuenta por los docentes (Jerez et al., 2016).

Hoy las instituciones de educación superior está conformada por 55 universidades, 25 de las cuales están dentro de las del Consejo de Rectores. Esta cifra se constituye por 16 de carácter estatal y 9 privadas. A la fecha, del total de universidades existentes en Chile, 46 han logrado acreditar y demostrar que cumplen con criterios de calidad. El promedio de años de acreditación llega a la mitad del máximo alcanzable (3,67 años), lo cual da un amplio margen de mejora en las instituciones de educación superior. Esto se agudiza, al ver que aún hay nueve instituciones (16\%) que no han logrado un nivel mínimo de calidad para dar cumplimiento a los estándares requeridos para la docencia de pre-grado y gestión institucional. Por lo tanto, 
aún en Chile hay temas pendientes referidos a los estándares mínimos exigidos por la normativa vigente para las universidades.

\section{Calidad y Buenas Prácticas en la docencia universitaria}

El análisis de la calidad de la docencia es un requisito importante para la educación superior. Este hecho se hace más relevante en un contexto donde hay una creciente demanda y diversidad de estudiantes, información asimétrica e incompleta y escasez de recursos públicos. (Pedraza-Rejas y Rodríguez-Ponce, 2015).

Existe una multiplicidad de conceptualizaciones para explicar lo que se entiende por calidad de educación. Dentro de ellas tomamos aquella que se indica como un proceso educativo de calidad que incluye la oportunidad de desarrollar un conjunto de habilidades y la adquisición de conocimientos que permita al sujeto/aprendiz mejorar su calidad de vida. Ambos hechos se evidencian, en que los estudiantes graduados tendrán más y mejores oportunidades de acceder a puestos de trabajo. (Akareem y Hossain, 2012). Por otro lado, Masse (1983) revela que la calidad de educación sería eficaz, en aquellas instituciones que reúnen las siguientes características en su proceso formativo: generan altas expectativas en sus estudiantes mediante la creación de un ambiente de apoyo académico y el desarrollo de habilidades básicas para la formación inicial. Estos ámbitos demuestran el esfuerzo institucional por mejorar el aprendizaje.

La literatura relacionada con la calidad en la educación, señala una diversidad de ámbitos a los cuales dedicar atención. Longanecker y Blanco (2003), plantean que la calidad de educación superior, estaría concentrada en el docente, dado que se debe considerar quién es la persona que enseña y por otra parte, cómo es que enseña. La organización de dos elementos, relator y metodología docente, incidirían en el logro de la calidad de la docencia. Wenger y Ferguson (2006), señalan que el conjunto de estrategias, procedimientos y técnicas metodológicas en la enseñanza, debiesen ser tratados en relación a la disciplina que se enseña. Los conceptos de calidad en la educación, involucran fundamentalmente, al equipo docente y sus estrategias de enseñanza-aprendizaje. En coincidencia con esto, Zabalza (2011) señala el imperativo de transitar desde una docencia centrada en la enseñanza hacia un estilo docente que comprometiera su quehacer con la calidad de los aprendizajes que se dan en el aula; y a la vez, involucrar el cambio ideológico en el rol del docente. Propone avanzar desde una concepción de docente como transmisor de información, hacia una concepción de gestor y generador de espacios de aprendizaje, es decir, transformar el aula en un lugar donde se activen los aprendizajes de los estudiantes. Es así como llegamos a la discusión actual de calidad de la educación vinculada con profesores universitarios y su práctica docente. (Jerez et al. 2012).

El término de buenas prácticas docentes tienes múltiples definiciones. Lo central es que se trata de un conjunto de acciones o estrategias que logran optimizar los procesos a través de los cuales los estudiantes mejoran su aprendizaje, independiente de sus condiciones de entrada. La esencia está puesta en el proceso, en un "hacer", en la acción desarrollada, no en las condiciones de partida ni en el resultado. Buenos resultados, no necesariamente son asimilables a buenas prácticas. Lo relevante de la investigación de BPD debe de considerar la identificación, selección y visibilidad de las mismas de manera de fortalecer la acumulación de evidencia que potencie la educación de calidad (Zabalza, 2012). Por su parte, Márquez (2002) entiende por buenas prácticas docentes las intervenciones educativas que facilitan el desarrollo de actividades de aprendizaje en las que se logren con eficiencia los objetivos formativos previstos y también otros aprendizajes de alto valor educativo.

Otro elemento a considerar para lograr una calidad en la enseñanza, es aprovechar el avanzado desarrollo de las tecnologías. Reig (2015) destaca la existencia de diferentes TIC's (Tecnologías de la Información y Comunicación) y las TAC's (Tecnologías del Aprendizaje y el Conocimiento). Estas herramientas pueden ser generadores de motivación y creatividad, además de desarrollar las habilidades multitarea de los actuales estudiantes. Ha quedado constatado, que las buenas prácticas, se relacionan directamente con el cambio metodológico en el proceso de enseñanza-aprendizaje, particularmente con la inclusión de las tecnologías, ajustado al nuevo perfil del estudiante. (Salaburu et al. 2011). Es así como tenemos que las BPD se refieren a las acciones "que explotan las nuevas herramientas tecnológicas para conseguir en el alumnado un aprendizaje autónomo que le motive a estar en permanente aprendizaje a lo largo de su vida"(Palomo et al., 2006). Centrando el análisis en los elementos de la teoría curricular y didáctica, las metodologías de la enseñanza son aquellos dispositivos que generan un potente y marcado impacto en la formación de los estudiantes. Al respecto, Zabalza (2003), propone cuatro dimensiones básicas en las metodologías de enseñanza-aprendizaje que favorecen una educación de calidad: la organización de los espacios y los tiempos, la orientación y gestión de las actividades de aprendizaje, las relaciones interpersonales, el modo de suministro de la información. En relación a los dos últimos, Tejada (2001) y Zabalza (2003) destacan que las estrategias didácticas deben contener a lo menos tres elementos básicos, la clase magistral, el trabajo en equipo y el trabajo autónomo del estudiante. De ser así, tal como lo 
proponen Mas y Olmos (2016), el docente de educación superior, debe contribuir en la mejora de los procesos de docencia, para lo cual es indispensable que el profesor desarrolle ciertas cualidades y atributos como la apertura al cambio, la reflexión, la capacidad crítica, la flexibilidad, entre otros.

En base a lo antes mencionado, este estudio se basa en las reflexiones de los actores centrales del proceso de enseñanza aprendizaje: docentes y estudiantes. Fernández y Torres (2011) mencionan las características que Epper y Bates (2004) consideran para catalogar a las buenas prácticas docentes. Entre ellas están el hecho que dichas prácticas contribuyen a mejorar el desempeño del proceso y su diseño considera un enfoque innovador. Indican que buenas prácticas las hace extrapolables a otros contextos. Por lo que una vez más, es importante el identificar, seleccionar y difundir a toda la comunidad estas acciones.

Las buenas prácticas docentes son hallazgos que permiten mejorar el proceso de enseñanza-aprendizaje en las aulas y con ello la calidad de la educación (Fernández y Torres, 2011). Finalmente, citando a Díaz et al. (2015), ellos indican que para el ámbito educativo las buenas prácticas educativas se configuran como saberes estratégicos dentro de la organización. Sobre esta mirada, es que se realiza un análisis reflexivo de los docentes en torno a sus estrategias de enseñanza-aprendizaje y de los estudiantes sobre su proceso formativo en el aula. En concreto, este estudio busca determinar aquellos factores que potencian el surgimiento de buenas prácticas docentes en dos carreras vinculadas al área de gestión en una universidad chilena. Para la clasificación de estos factores se toma la indicada por Zabalza (2003). Sin embargo, es necesario indicar que los aspectos se interrelacionan y pueden ser parte de más de una clasificación.

\section{METODOLOGÍA}

La investigación realizada es de tipo cualitativo. La forma de aproximación fue de manera inductiva. El trabajo de campo consistió en recoger las opiniones y reflexiones sobre las prácticas docentes en el aula desde la perspectiva de los sujetos (estudiantes y docentes) para identificar los factores que inciden en el surgimiento de buenas prácticas docentes. Se indagó en los aspectos metodológicos como representaciones válidas de la calidad universitaria y al mismo tiempo, se centró en los factores que pueden favorecen o no, las prácticas en el aula como por ejemplo: rasgos interpersonales e intrapersonales, así como la forma y modo de instalar las actividades de enseñanza. La clasificación de los factores determinados se hace de acuerdo a las cuatro dimensiones propuestas por Zabalza (2003): el modo de suministro de la información, las relaciones interpersonales, la orientación y gestión de las actividades de aprendizaje y la organización de los espacios y los tiempos. Los resultados y discusión que se proponen en este trabajo sólo se refieren a los hallazgos de los grupos focales de docentes y estudiantes.

El estudio se hizo con docentes y estudiantes de las carreras de Ingeniería Comercial e Ingeniería en Información y Control de Gestión de la Escuela de Ciencias Empresariales de la Universidad Católica del Norte. Los profesores participantes fueron seleccionados dentro del total de profesores que hicieron clases durante el año 2015. El estudio incluyó a seis docentes con un nivel de satisfacción en la encuesta docente (evaluación docente) por sobre el $90 \%$ promedio en las cinco dimensiones evaluadas por el instrumento, siguiendo una de las líneas definidas por Zabalza (2012). Para el caso de los estudiantes la muestra la conformaron ocho estudiantes de la carrera de Ingeniería de la Información y Control de la Gestión, nueve estudiantes de la carrera de Ingeniería Comercial. En ambos casos (docentes y estudiantes) la selección de participantes fue por conveniencia o intencional. Para el caso de los estudiantes, se requirió la participación de estudiantes de diferentes niveles del programa de formación y además, que el grupo estuviese constituido, tanto por hombres como por mujeres. En el caso de los profesores, consideró que participaran docentes de todos los niveles del programa de formación, y también que haya una representación tanto de hombres como mujeres. El grupo de docentes realizó clases a los estudiantes de la muestra durante algún momento de su formación.

La técnica usada para la recogida de información fue grupo focal, por cuanto, es uno de los procedimientos del ámbito social, que utiliza el ejercicio de pregunta y respuesta, para generar un diálogo. Tal como lo sugieren Taylor y Bogdan (2002), los fenómenos se deben entender desde la perspectiva del actor, es decir, como experimenta el mundo de sucesos, la percepción de lo más importante para las personas. Desde allí que sea esencialmente humanista, por lo tanto lo metodológico cuida que esa "fotografía" sea lo más parecida a la realidad posible. Asimismo, como lo plantea Gordo (2008), con esta técnica se generan disputas de manera organizada y guiadas desde experiencias personales. Por lo tanto, dada la problemática abordada, esta práctica de discusión favoreció el ambiente de conversación. Se realizaron tres (03) grupos. Uno (1) de ellos de profesores y dos (2) grupos de estudiante. Los dos (2) grupos de estudiantes, se dio básicamente porque al interior de la escuela se imparten dos (2) carreras y eso permitió un ambiente de confianza en temáticas concernientes a cada programa de formación. Obtener la opinión de docentes y estudiantes enriqueció la información y al mismo tiempo, permitió la saturación. La duración de cada encuentro fue aproximadamente de 60 a 80 minutos y las conversaciones fueron grabadas audiofónicamente, en su totalidad. Para su realización se solicitó, la firma del consentimiento informado de 
los participantes. Cada uno de los textos analizados, fue tratado con la técnica de análisis de contenido y reportó un informe con el análisis, interpretación y conclusiones. Estos resultados fueron compartidos con los sujetos de la muestra.

El grupo docente, representó a lo menos doce (12) asignaturas en las carreras de la escuela, entre ellas, áreas de administración, marketing, sistemas de información, macroeconomía, microeconomía y desarrollo de actitud emprendedora. Finalmente para el levantamiento de las reflexiones sobre las prácticas docentes, se consideraron las categorías o factores que favorecen las buenas prácticas en el aula, a la luz de las conversaciones en los grupos focales, luego de su análisis de contenido.

\section{RESULTADOS}

En la conversación con docentes y estudiantes, surgen los siguientes factores que favorecen el desarrollo de las buenas prácticas. El primer factor es la Fisonomía. En los grupos consultados, arrojó un rasgo común: el de la edad. Docentes como estudiantes, están dentro del rango de personas jóvenes. En los grupos de estudiantes, aparece una edad promedio de los veinte y dos años mientras que los docentes con una edad promedio de treinta y dos años. Los estudiantes cursaban el quinto $\left(5^{\circ}\right)$ y sexto $\left(6^{\circ}\right)$ nivel del plan de formación correspondiente a cada carrera, es decir con una permanencia de tres (3) a cuatro (4) años de estudios en la institución. Esta audiencia estuvo conformada por más mujeres que hombres. Por otra parte, los docentes; bordearon entre dos (2) y tres (3) años de experiencia docente, donde la mayoría había estado trabajando en docencia, dos (2) años, en promedio.

Relacionado con lo anterior, emerge el segundo factor, definido es Afinidad por la docencia, donde los docentes manifestaron su acercamiento a la docencia por tres (3) grandes núcleos: a) Entorno Familiar: Los docentes relataron historias cercanas, en la familia, que los llevaron a tener experiencias directas con la enseñanza; por lo tanto desde temprana edad, ellos estuvieron expuestos a cuestiones de la enseñanza. $b$ ) Entorno Social: El grupo indicó, cómo el medio próximo como la escuela, amistades u otros, incidieron a la hora de su preferencia por la docencia. En este sentido, señalaron que siempre ellos eran los que explicaban materias en la escuela, por ejemplo, a sus compañeros o amigos. c) Modelos de referencia: Los docentes explicaron, sobre cómo ellos fueron "enseñados", las formas que usaban sus profesores, tanto los "buenos" y "malos" profesores en su formación escolar y universitaria. Es así, como surgió el concepto de modelo de referencia, en tanto, algunos fueron provocados por aquellos "malos" profesores a no realizar acciones de aquella naturaleza y querer superarlos, convirtiéndose ellos, en profesores de "buenas prácticas".

Luego, aparece el tercer factor sobre Metodologías y Estrategias en el aula, tanto en docentes como estudiantes. Los hallazgos señalan que ésta se divide en dos ámbitos; uno referido a la esfera técnica y otro, referido a la esfera personal.

En el primero de ellos, esfera técnica emergen: a) La importancia del trabajo en equipo o grupal por cuanto creen necesaria esa oportunidad, como instancia de aprendizaje entre pares (percepción de los estudiantes) y además, el desarrollo de otras habilidades distintas a las disciplinares (percepción de los docentes). b) La importancia de ejemplificar con casos reales y no ficticios o sacados de libros, y traer siempre experiencias laborales referidas a las temáticas abordadas en la clase (percepción de los estudiantes) y casos de contingencia nacional e internacional (percepción de los docentes). c) El uso permanente y acotado de las tecnologías de la información y comunicación, dado que es motivador e incentivador en el acercamiento al aprendizaje mediante, por ejemplo, el uso las redes sociales, videos, películas por citar algunos (percepción significativa de docentes y estudiantes). d) El uso de un lenguaje amigable, concreto y práctico en el aula (percepción de los estudiantes), un lenguaje contemporáneo que permita vincularse con los estudiantes desde su cotidiano, sin extinguir el lenguaje técnico de su profesión (percepción de los docentes). e) La utilidad de los trabajos de aplicación práctica (percepción de los estudiantes) y además la realización de ejercicios o tareas en conjunto con el profesor (percepción de docentes y estudiantes). f) La importancia de las salidas a terreno 0 visitas a seminarios, en donde se puedan enfrentar a otros escenarios de aprendizaje, que les permita confrontar lo abordado en clases o lo leído en textos. Además los grupos señalan, que el docente debe tener dominio de la disciplina que enseñan y además organizar de modo tal las clases que el tiempo sea una oportunidad para el aprendizaje y no un obstaculizador, provocando un tratamiento rápido y poco profundo o muchos contenidos en poco tiempo.

En el segundo de ellos, esfera personal, emergen cuestiones, la mayoría citada por los estudiantes, tales como: a) La relevancia de la paciencia ejercida por el docente, a la hora de practicar la enseñanza, por cuanto, esto incidirá directamente en el aprendizaje de los estudiantes. b) Valor por la capacidad de motivación e incentivación que muestra el docente en lo que enseña, que atiende a la estimulación cognitiva de sus aprendices. c) La importancia de la participación en clases de todos los estudiantes, ligada 
a la anterior, que permite la movilización de ciertas habilidades dispuestas para el aprendizaje significativo y efectivo. d) Valor a la capacidad del docente de vincularse emocional y afectivamente con sus estudiantes, capacidad del docente de conocer a sus estudiantes, desde saber sus nombres hasta contener 0 comprender problemáticas que a veces ellos enfrentan.

El cuarto factor que surge es el de Aprendizajes, referido a tres elementos coincidentes en docentes como estudiantes. El primero de ellos, apunta a que el aprendizaje siempre sucederá de manera profunda y significativa, en la medida que sea práctico, con oportunidades de aplicabilidad de conocimientos y no memorísticos. Los estudiantes refirieron a la práctica profesional como instancia de consolidación del aprendizaje, es decir, el contacto con la realidad laboral es sustancial para el aprendizaje. En tanto, los docentes explicaron que el aprendizaje real, está expresado en la capacidad cognitiva de los estudiantes para resolver problemas simples y complejos de la profesión. El segundo elemento coincidente, es la motivación personal, tanto en estudiantes como en profesores, sin lo cual cualquier esfuerzo técnico en desarrollar contenidos, no es suficiente. Los estudiantes asocian la motivación de los docentes por realizar su clase, en la didáctica que usan, es decir si es motivadora, participativa, innovadora, etc. para poder conseguir aprendizajes. El tercer y último elemento, que se manifestó, es el espacio para explicar a otros ciertos contenidos, es decir, estrategias ligadas a exposiciones orales frente a una comisión, trabajo en grupo por ejemplo; en donde se pone en juego el conocimiento adquirido y su transferencia eficaz. Los docentes al respecto, indicaron dos ámbitos referidos al aprendizaje. Uno de ellos se relaciona con las habilidades cognitivas que ellos pretenden desarrollar en sus estudiantes cada vez que entran al aula, por ejemplo, el desarrollo de la comprensión y análisis de problemas de la profesión, cuestionamiento o pensamiento reflexivo permanente, generación y utilización de lenguaje técnico acorde al nivel de formación. El otro de ellos, es el desarrollo de actitudes, tales como, habilidades sociales, autonomía, uso de error y su aceptación, generación de expectativas.

En el transcurso de las intervenciones, se logró levantar y evidenciar el quinto factor relevante, el Profesor de excelencia. Los estudiantes en este aspecto, indicaron a lo menos catorce docentes que se distinguen del resto, acercándose al $33 \%$ de la población académica de la escuela, de los cuales tres de ellos son extranjeros y nombran a dos que ya no se encuentran dictando asignaturas en la carrera, cuestión que entrevé, el impacto o significancia de su paso por su formación. Por su parte, los docentes señalaron que su rol en el aprendizaje de sus estudiantes era, un generador de motivación extrínseca, un profesional sin egos, un docente que domina la disciplina que enseña, un docente empático, un docente que se hace cargo de su mejora continua y es responsable de hacer seguimiento del aprendizaje de sus estudiantes. En su conjunto, los estudiantes concordaron, con los nombres de los docentes, entrevistados y por su parte el grupo de profesores, cualificaron coincidentemente, una serie de habilidades o capacidades que los estudiantes nombraron, tales como, capacidad para ponerse en el lugar de otros, capacidad de auto-perfeccionamiento, capacidad de responsabilizarse por el aprendizaje de sus estudiantes, por nombrar algunos.

Luego, emerge el sexto factor Sugerencias de Mejora, en que los estudiantes y docentes concuerdan con dos elementos. El primero de ellos, es la necesidad de trabajar en el aula con el conocimiento aplicado, contextualizado a la realidad laboral que los estudiantes enfrentarán, sumándose a ello, proyectos acotados, salidas a terreno para comprender la realidad y actividades que integren todas las asignaturas del semestre y nivel. Lo anterior, hace surgir el segundo elemento, que es la adquisición de herramientas didácticopedagógicas para todos los profesores, para implementar efectivamente el aprendizaje del nuevo conocimiento. Para los profesores consultados, aún sigue siendo una problemática la forma de trabajar con estudiantes de primer año, aun cuando han aplicado varias estrategias, creen necesario, poder abordarlo de manera distinta que a otros niveles. Los estudiantes, creen necesario implementar un seguimiento a los docentes en el aula y que la rotación y renovación docente, sea efectiva.

Toda la información tratada con anterioridad, tiene una alta concomitancia en los grupos de estudiantes y docentes consultados. No obstante, también existieron elementos, distintos en cada grupo consultado. En este sentido, los docentes expresaron como factor propio la Valoración y estrategias diferenciales en el aula, es decir, por una parte el conocimiento que ellos tienen sobre cuanto son valorados por sus estudiantes; cuestión que toman con humildad y por otra parte, la preocupación que ellos demuestran por los aprendizajes que no son conseguidos, cuestión que expresan como, hacer un alto en el proceso e insistir en los nuevos conocimientos, disponiéndolos de una manera distinta. En otro sentido, una diferencia en el caso de los estudiantes, plantean como factor propio el Ideal de clase que supuso, tres, niveles, que en su conjunto expondría una clase ejemplar. El primero de ellos, son técnicas didácticas, como una clase que contenga ejemplificación permanente, el nivel de explicaciones con un lenguaje claro y simple, más no réplicas de textos. El segundo de ellos, es lo administrativo, es decir, una cantidad de estudiantes adecuado en el aula y un docente con dominio de la disciplina que enseña. El último nivel es, las características personales, como un docente cercano a sus estudiantes, interesado y preocupado por lo que les ocurre, cuestión que permitiría un vínculo emocional entre ellos. 
En resumen, se tienen ocho factores que favorecen las BPD de educación superior en el caso de dos carreras del área de gestión en la Universidad Católica del Norte. Seis de ellos son manifestados tanto en docentes como estudiantes: Factor $\mathrm{N}^{\circ}$ 1: Fisonomía; Factor $\mathrm{N}^{\circ}$ 2: Afinidad por la docencia; Factor $\mathrm{N}^{\circ} 3$ : Metodologías y estrategias en el aula; Factor 4: Aprendizajes; Factor $N^{\circ}$ 5: Profesor de Excelencia y Factor № 6: Sugerencias de mejora. Además se tienen un factor propio de los docentes, denominado Valoración y estrategias diferenciales en el aula y un factor propio de los estudiantes definido como: Ideal de Clase.

\section{DISCUSIÓN}

Los rasgos docentes, fisonomía, incidirían en la mejora de su propia práctica. En este aspecto, es necesario destacar que los docentes han tenido alguna vinculación a la docencia desde temprana edad, ya sea a nivel familiar o con el entorno, cuestión que determinaría su afinidad o preferencia por la docencia, sin descuidar su desarrollo disciplinar. En tanto, los estudiantes, conservan una dialéctica similar a la de aquellos profesores distinguidos, es decir, la fisonomía de todos los grupos, repara en un hecho generacional, que acercaría las brechas entre docentes y estudiantes. Esto se puede apreciar por el uso del lenguaje, afanes, tecnología y otros aspectos, que en su conjunto, ven incrementado el aprendizaje efectivo de sus estudiantes.

De acuerdo con lo planteado por Rubio et al. (2016) es necesario que aquellos que ejercen la docencia, demuestren un nivel de reflexión crítica sobre su propia práctica, tendiente a la mejora continua, porque existirían aprendizajes de calidad los que serían bien valorados por los estudiantes. Es importante el ejercicio realizado, porque sienta un precedente sobre la actuación reflexiva de los profesores y estudiantes, que participaron en el estudio.

En otro aspecto, las condiciones por las cuales existe una afinidad por la docencia, genera creatividad (cuasi innata en los docentes) y originalidad en las formas y modos de intervención didáctica. Se detectó que los docentes tuvieron una exposición docente en sus familias y entorno cercano. Según Denzin (1989) citado en Leite (2011), las historias de vida, marcan fuertemente las teorías implícitas en los sujetos, transfiriendo aquella naturaleza en el ejercicio de la profesión o rol que ejerzan. Consecuentemente, el rol docente, asociado a las metodologías de clases, referidas a cualidades personales y a dominio didáctico, incide directamente en el desarrollo de buenas prácticas docentes. Estos hallazgos se relacionan con los resultados enumerados por Bain (2006) para los mejores profesores. El concepto que manejan los estudiantes sobre lo que es ideal de clase promueve aprendizajes de naturaleza profunda. En este sentido, Tejada (2001), sostiene que promoviendo el trabajo colaborativo, organizando la enseñanza en función de las competencias, con mejores didácticas, incrementará las herramientas de aprendizaje autónomo y permanente, en los estudiantes.

Los hallazgos de este estudio ratifican el hecho que las buenas prácticas docentes, están en directa relación con las metodologías de una clase de calidad, exhibiendo un docente con dominio disciplinar, con intervenciones pedagógicas de alto nivel y capaz de generar una vinculación emocional con otros. Desde el punto de vista estudiantil, la ascendencia que demuestra un docente de excelencia, se refiere casi exclusivamente a los aspectos personales, atribuido a la cercanía que el docente puede establecer con sus estudiantes, a la capacidad de vinculación emocional y de confianza. Resultados coincidentes con los de Fernández y Torres (2015), quienes señalan que entre los principales factores para proporcionar buenas prácticas docentes con apoyo de TIC se encuentran: fomentar la motivación hacia su uso; favorecer el trabajo personalizado; favorecer la comunicación con los estudiantes y estimular el aprendizaje o la adquisición de conocimientos.

Las buenas prácticas descritas por los docentes y estudiantes establecen una relación de simetría y de interdependencia en los niveles analizados, porque cada una de las categorías conlleva a otra y retroalimenta su constitución. Se verifica lo indicado por Zabalza (2012), las buenas prácticas docentes intencionan aprendizajes profundos y significativos en los estudiantes, además de motivación, efectividad en las actividades y construcción de identidad profesional. Además existe una interdependencia positiva entre una buena práctica docente para generar estrategias innovadoras en el aula y mejorar la calidad en la educación. Estos resultados son coincidentes con los encontrados por Fernández et al. (2012), quienes indican que los profesores que estudiaron tienden a utilizar estrategias para implicar y retar a sus estudiantes.

Desde esta perspectiva se deben comprender las buenas prácticas docentes como un ente dinámico y vivo, cuya organización descansa en el sujeto del profesor acompañado por las condiciones mínimas de operación de su praxis (Zabalza, 2012). En la línea de Vásquez (2015) resulta imprescindible promover apoyos y estímulos a los docentes para el desarrollo de unas tareas cada vez más diversas y complejas. Asimismo, se hace necesario considerar, el desarrollo de competencias docentes para poder enfrentar los 
desafíos, del nuevo escenario universitario. Una forma de potenciar innovación en el aula y desarrollar métodos centrados en el aprendizajes significativos (Gargallo et al. 2015) es mediante la difusión de buenas prácticas docentes y de establecer las condiciones necesarias para que estas puedan surgir 0 potenciarse, tal como lo señalan Mas y Olmos (2016). Aquí es cuando estudios como el descrito en este manuscrito cobran relevancia para difundir los factores que potencian las buenas prácticas docentes. 
Para finalizar y de acuerdo a lo indicado anteriormente, los ocho factores encontrados como determinantes para el desarrollo de buenas prácticas docentes, se clasifican de acuerdo al enfoque de Zabalza (2003). A su propuesta se le agregan las relaciones intrapersonales. En esta dimensión se encuentran dos factores: Fisionomía y Afinidad por la docencia. En la categoría modo de suministro de información, es donde más factores se encuentran. En total cinco factores se pueden incluir aquí. Finalmente el factor denominado aprendizajes es el vinculado a la tercera dimensión de Zabalza. La última dimensión no fue materia de análisis en la presente investigación. El factor Intrapersonal no corresponde a Zabalza (2003). Fue hallazgo de esta investigación y se agregó por la afinidad a los constructos de Relaciones interpersonales. Todo esto se visualiza en la Tabla 1.

Tabla 1: Clasificación de Factores Determinantes de Buenas Prácticas Docentes

\begin{tabular}{|l|l|}
\hline \multicolumn{1}{|c|}{ Clasificación Zabalza (2003) } & \multicolumn{1}{c|}{ Factores Identificados } \\
\hline $\begin{array}{l}\text { Relaciones interpersonales - } \\
\text { intrapersonales }\end{array}$ & $\begin{array}{l}\text { Factor } \mathrm{N}^{\circ} 1: \text { Fisonomía } \\
\text { Factor } \mathrm{N}^{\circ} 2: \text { Afinidad por la docencia }\end{array}$ \\
\hline Modo suministro de información & $\begin{array}{l}\text { Factor } \mathrm{N}^{\circ} \text { 3. Metodologías y estrategias en el aula } \\
\text { Factor } \mathrm{N}^{\circ} 5: \text { Profesor de Excelencia } \\
\text { Factor } \mathrm{N}^{\circ} \text { 6: Sugerencias de mejora } \\
\text { Factor propio Estudiantes: Ideal de Clase } \\
\text { Factor propio Docentes: Valoración y estrategias diferenciales en el aula }\end{array}$ \\
\hline $\begin{array}{l}\text { Orientación y gestión de las actividades } \\
\text { de aprendizaje }\end{array}$ & Factor $\mathrm{N}^{\circ} 4:$ Aprendizajes \\
\hline $\begin{array}{l}\text { Organización de los espacios y los } \\
\text { tiempos. }\end{array}$ & No se analizó \\
\hline
\end{tabular}

Estos resultados permiten ampliar o adecuar las dimensiones de calidad en la educación de Zabalza (2003) al ámbito de educación superior de estudiantes del área de gestión de empresas en Chile. De acuerdo a estudios en la línea de BPD, resulta relevante seguir en la línea de identificar, analizar y difundir las buenas prácticas docentes para continuar en la línea de mejoramiento permanente de la calidad en la educación.

\section{CONCLUSIONES}

De acuerdo al estudio reflexivo realizado a un total de seis profesores nóveles con excelente evaluación docente y 18 estudiantes del área de gestión de organizaciones, se pudo determinar ocho factores que favorecen el desarrollo de buenas prácticas docentes. Estos factores se clasificaron siguiendo las dimensiones referidas a la calidad en la educación superior propuestas por Zabalza (2003).

En base a los resultados, sólo para este contexto, se puede inferir que la forma de realizar la docencia, es decir las estrategias docentes y las características de los profesores en el aula, se convierten en los principales factores para potenciar las BPD y con ello la calidad en la educación. En consecuencia, la principal dimensión es la denominada Modo de suministro de información ya que obtuvo la mayor cantidad de factores (cinco de ocho). Los cinco factores que le conciernen a esa dimensión son: Factor $\mathrm{N}^{\circ} 3$ : Metodologías y estrategias en el aula; Factor $\mathrm{N}^{\circ}$ 5: Profesor de Excelencia y Factor $\mathrm{N}^{\circ}$ 6: Sugerencias de mejora; factor propio de los docentes, denominado Valoración y estrategias diferenciales en el aula y factor propio de los estudiantes definido como: Ideal de Clase. La categoría Relaciones Interpersonales e intrapersonales logró el Factor $N^{\circ}$ 1: Fisonomía y Factor $N^{\circ} 2$ : Afinidad por la docencia. En este sentido, resulta importante realzar la dimensión no estudiada por Zabalza (2003) que es la de Relaciones/Aspectos Intrapersonales, que se reveló con el presente estudio, donde aparecen cualidades personales de los docentes que se manifestarían en el aula. Finalmente la dimensión Orientación y Gestión de las actividades, incluye el Factor 4: Aprendizajes.

Cabe indicar la coincidencia de elementos claves para la mejora expuesta en las conversaciones con profesores y estudiantes. Con estos resultados se aprecia una interesante y compleja interdependencia de los atributos y cualidades. Por ello los factores se agrupan en una sola dimensión, sin embargo se entiende que existe una interdependencia entre varios de ellos.

Para el caso de los docentes, destacan sus experiencias iniciales con la docencia que les hizo no repetir errores vividos en su formación y su permanente interés por innovar en su docencia. En el caso de los 
estudiantes, es trascendental para ellos y para sus aprendizajes, dos elementos claves: metodologías experimentadas en el aula y el perfil del profesor.

\section{REFERENCIAS}

Akareem, H. y Hossain, S. Perception of Education Quality in Private Universities of Bangladesh: A Study From Students' Perspective, Disponible en: https://goo.gl/AHzdbW, Journal of Marketing for Higher Education, 22(1), 11-33 (2012)

Bain, K. Lo que hacen los mejores profesores universitarios, Valencia, PUV (2006)

Díaz, M., Borges, M., Valadez, M. y Zambrano, M. Valoración de buenas prácticas docentes a través de observación sistemática, Disponible en: https://goo.gl/NccKsa, Universitas Psychologica, 14(33), 913-922 (2015)

Fernández, J.M. y Torres, J.A. Actitudes docentes y buenas prácticas con TIC del profesorado de Educación Permanente de Adultos en Andalucía, Revista Complutense de Educación, 26 Núm. Especial, 33-49 (2015)

Fernández, A., Maiques, J. y Galcerá, A. Las buenas prácticas docentes de los profesores universitarios: estudio de casos, Revista de Docencia Universitaria, 10(1), 105-122 (2012)

Gargallo, B., Garfella, P., Sahuquillo, P., Verde, I., Jiménez, M.A. Métodos centrados en el aprendizaje, estrategias y enfoques de aprendizaje en estudiantes universitarios, doi: 10.4438/1988-592X-RE-2015-370304, Revista de Educación, 370, 229-254 (2015)

Gordo, A. y Serrano, A. (Coords.) Estrategias y Prácticas Cualitativas de Investigación Social, Disponible en: https://goo.gl/NGCXst, Madrid, Pearson Educación (2008)

Jerez, O., Orsini, C. y Hasbún, B. Atributos de una docencia de calidad en la educación superior: una revisión sistemática, Estudios Pedagógicos XLII (3), 483-506 (2016)

Leite, A. Historias de Vida de Maestros y Maestras. La Interminable Construcción de las Identidades: Vida Personal, Trabajo y Desarrollo Profesional. Tesis Doctoral, Facultad de Ciencias de la Educación, Universidad de Málaga, España (2011)

Longanecker, D. y Blanco, C. Student Financial Assistance, Student Success: Statewide P-16 Systems, State Higher Education Executive Officers (SHEE0), (2003)

Mas, O. y Olmos, P. El Profesor Universitario en el Espacio Europeo de Educación Superior. La Autopercepción de sus Competencias Docentes Actuales y Orientaciones para su Formación Pedagógica, Revista mexicana de investigación educativa, 21 (69), 437-470 (2016)

Masse, B. Quality Control in Education, The Clearing House, 57(2), 66-68 (1983)

Pedraza-Rejas, L. y E. Rodríguez-Ponce. El aseguramiento de la calidad: un imperativo estratégico en la educación universitaria. Disponible en: https://goo.gl/jpQJEv, Ingeniare. Revista Chilena de Ingeniería, 23(1), 4-5 (2015)

Reig, D. TIC, TAC TEP y sus bases en un video de animación. El Caparazón Blog. Video en línea, Disponible en: https://goo.gl/XswfxB (2015)

Rubio, M.J., Ruiz, A., y Martínez-Olmo, F. Percepción Del Alumnado Sobre la Utilidad de las Actividades de Aprendizaje para Desarrollar Competencias, Disponible en: https://goo.gl/kuJRBd, Revista de Investigación Educativa, 34(1), 221-240 (2016)

Salaburu, P., Haug, G. y Mora, J. G. España y el Proceso de Bolonia, Un Encuentro Imprescindible. Madrid: Academia Europea de Ciencias y Artes, Disponible en: http://goo.gl/wbHgpM (2011)

SIES. Informe Nacional de Antecedentes. El Aseguramiento de la Calidad de la Educación Superior en Chile, Comité de Coordinación. Sistema Nacional de Aseguramiento de la Calidad de la Educación Superior en Chile (SINAC-ES), Santiago de Chile (2012) 
Taylor, S.J. y Bogdan, R. Introducción a los métodos cualitativos de Investigación. La búsqueda de significados, Editorial Paidós, Barcelona (2002)

Tejada, J. Planificación de Acciones de Formación Ocupacional: Estrategias Metodológicas. Documento Mimeografiado, Dpto. de Pedagogía y Didáctica, Universidad Autónoma de Barcelona, España (2001)

Vásquez, J. A. Nuevos Escenarios y Tendencias Universitarias. Disponible en: https://goo.gl/HxPCzb, Revista de Investigación Educativa, 33(1), 13-26, (2015)

Wenger, M., Ferguson, C. A Learning Ecology Model for Blended Learning from Sun Microsoftsystems. (Chapter 6) En C.J. Bonk, C. R. Graham (Eds.). Handbook of blended learning: Global Perspectives, local designs. Pfeiffer Publishing, San Francisco, EE.UU. (2006)

Zabalza, M.A. y Zabalza-Beraza, M.A. Competencias Docentes del Profesorado Universitario. Narcea Ediciones, Madrid, España (2003)

Zabalza, M. A. Metodología Docente, Revista de Docencia Universitaria, 9(3), 75-98 (2011)

Zabalza, M.A. El estudio de las "buenas prácticas" docentes en la enseñanza universitaria, Revista de Docencia Universitaria, 10 (1), 17-42 (2012) 